The Journal of International Trade \& Economic Development

An International and Comparative Review

ISSN: 0963-8199 (Print) 1469-9559 (Online) Journal homepage: http://www.tandfonline.com/loi/rjte20

\title{
Product-level estimation of import demand: Simulating the effects of tariff harmonisation
}

\section{Winston Moore \& Diego Morris}

To cite this article: Winston Moore \& Diego Morris (2012) Product-level estimation of import demand: Simulating the effects of tariff harmonisation, The Journal of International Trade \& Economic Development, 21:5, 655-676, DOI: 10.1080/09638199.2010.514938

To link to this article: https://doi.org/10.1080/09638199.2010.514938

曲 Published online: 23 May 2011.

Submit your article to this journal $\pi$

Џlll Article views: 152 


\title{
Product-level estimation of import demand: Simulating the effects of tariff harmonisation
}

\author{
Winston Moore* and Diego Morris \\ University of the West Indies, Cave Hill Campus, Department of Economics, \\ Bridgetown, Barbados
}

(Received 3 February 2010; final version received 9 August 2010)

\begin{abstract}
Developing countries have traditionally used import tariffs to protect infant industries and raise revenues to finance government expenditure plans. This approach, however, has tended to protect inefficient industries and to some extent hindered economic development. A disaggregated import demand model is estimated using monthly observations on 91 of the most frequently imported product items in Barbados. The results are then employed to evaluate the feasibility of harmonising tariff rates to some single rate across product categories. The results suggest that the estimation of aggregate import demand equations is not accepted by the data and therefore could result in misleading inferences. The policy simulation exercise indicates that a single applied tariff at the $30 \%$ level would essentially be revenue neutral, while rates above this level would lead to reductions in tax receipts.
\end{abstract}

Keywords: imports; panel data; price elasticity; tariffs; trade reforms

\section{Introduction}

Imports are a key component of all Caribbean economies. Given the limited availability of natural resources in the Caribbean, imports are fundamental inputs for regional industries, particularly tourism. Imports can also positively affect economic growth if import productivity increases the rate of investment in domestic capital relative to the import intensive industries (James 2006). Figure 1 plots the ratio of imports of goods and services to GDP for 14 Caribbean territories in 2005. On average, imports of goods and services represent about $64 \%$ of GDP, or about 37 percentage points higher than the average for the rest of the world. In Guyana, the ratio reached as high as $124 \%$, with most of the other countries falling in the $60 \%$ to $80 \%$ of GDP range.

\footnotetext{
*Corresponding author. Email: winston.moore@cavehill.uwi.edu 


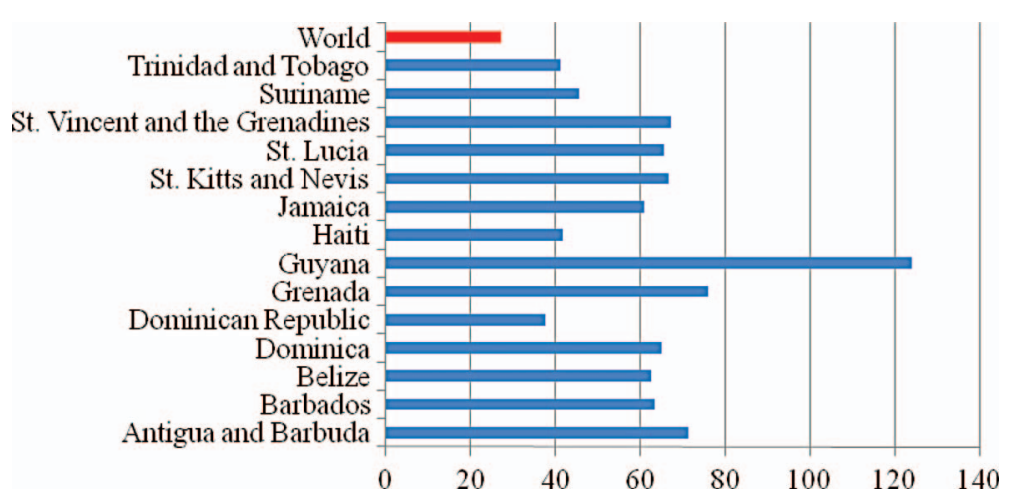

Figure 1. Imports of goods and services ( $\%$ of GDP, 2005). Source: World Development Indicators Online.

Much of the previous literature on import demand in the Caribbean and abroad has been either country-specific or looked at import demand for particular regions (see Deyak et al. 1989 for a review of the literature for the United States and other developed countries). Moreover, this interaction has tended to focus on employing differing econometric techniques to estimate aggregate demand functions. Building on this literature, this study attempts to provide a product-level assessment of import demand in Barbados using monthly observations on 91 products at the four-digit Standard International Trade Classification (SITC) level between January 2000 and September 2008.

There is good reason to think that import demand should be assessed at the product level rather than at the aggregate level. This is most evidenced by the fact that many previous studies have found that estimates of aggregate import demand have been characterised by parameter instability (Mah 1993; Zietz and Pemberton 1993; Bahmani-Oskooee and Rhee 1997). It is very possible that this parameter instability could be driven by changes in the underlying import demand for particular products or the inclusion of new products in the individual's consumption bundle.

Product-level estimates of import demand are particularly useful for policy purposes. Trade negotiators may utilise the estimates provided in this study to conduct simulations of the potential impact of tariff changes. The study's findings may also be employed to illustrate the countries or region(s) with which there is greater need for negotiations as they may represent source markets for respective goods. Fiscal authorities may also find the estimates useful for forecasting future trade tax receipts as well as the effects of tariff harmonisation, i.e. a single tariff rate applied to all imported products.

Given the importance of imports to regional economies, it is important to understand the factors that influence import demand in the Caribbean. 
Gafar (1995) estimated income elasticities for three Caribbean countries (Guyana, Jamaica and Trinidad and Tobago) and found that relative prices and real income were the most essential determinants of aggregate import growth in these territories. Similar results were obtained by Maxwell and Moore (2004) using panel cointegration techniques and annual observations on 12 Caribbean countries. Birchwood and Jhinkoo (2007) tested the hypothesis of James (2006) that there is a link between economic development and the elasticity of demand of imports. The authors reported that in most countries the income elasticity coefficient fell below unity, suggesting that the demand for imports is inelastic.

The current study is most closely related to Shiells (1991) which estimates disaggregated import demand functions for the United States to test the hypothesis that errors may result from using unit-value indexes as measures of import prices. In the study, the quantity of imports demanded for a given commodity group is regressed on current as well as lagged prices of imports and competing domestic goods, economic output and quarterly dummy variables to account for seasonality. The lags of imports and output are assumed to follow a gamma distribution. Estimating the model using autoregressive two-stage least-squares, Shiells reports that for most commodity groups the import price elasticity was negative and greater than one, while the output elasticity varied from commodity to commodity. Overall, the study suggested that using unit-value indices did not introduce significant bias into the regression results. The present study differs from Shiells (1991) in three main areas: (1) the model explicitly considers the effects of import duties, rather than subsuming them in import prices; (2) a panel estimation approach is employed, which allows the author to consider both the space and time dimensions; and (3) the results are utilised to consider the potential implications of tariff harmonisation.

The remainder of this article is structured accordingly: following the introduction, Section 2 outlines the methodological strategy employed to estimate the disaggregated import demand functions, while Section 3 offers an assessment of the product-level trends in imports in Barbados. Section 4 provides a discussion of the findings of the article. Finally, Section 5 summarises the main policy implications and concludes.

\section{Econometric strategy}

If there are $i=1,2, \ldots, k$ products, traditional import demand equations usually relate real imports $(m)$ to real income $(y)$ and the ratio of import prices $\left(P_{\mathrm{m}}\right)$ to domestic prices $\left(P_{\mathrm{d}}\right)$. Estimating a traditional demand function for imports using this approach can be somewhat difficult due to the interaction between supply and demand. Murray and Ginman (1976), therefore, note that this problem has been traditionally solved by assuming that supply elasticity is infinite: an almost unlimited supply of a product or 
service at a given price. Such an assumption is likely to hold if one is dealing with small open economy such as Barbados, which given its size is a price taker. Assuming a log-linear specification, this study therefore estimates the import demand function as:

$$
m_{i t}=\alpha_{i}+\beta_{i} y_{i t}+\gamma_{i}\left(\frac{p_{m}}{p_{d}}\right)+\phi_{i} d_{i t}+\varepsilon_{i t}
$$

where, $\beta$ and $\gamma$ are the income and relative prices elasticities of demand, respectively, $\phi$ is the elasticity of demand with respect to duties applied, $d_{i t}$ represents the duties applied on each product, while $\varepsilon$ is an error term, which is assumed to be normally distributed with a zero mean and a constant variance. All estimated equations include seasonal dummy variables as well as an AR term. Equation (1) is estimated for each of the 91 products under the assumption that expenditure shares are constant fractions of total expenditure.

The model estimated above allows one to compare the responsiveness of various commodities to changes in income, prices and duties. It is expected that non-durable products, in general, should be less responsive to changes in both prices and income as theory suggest that the greater the percentage of income spent on a commodity, the greater its elasticity is likely to be. Because most durable items tend to represent a large proportion of the consumer's budget, the price elasticity of demand for these items should, on average, be higher than those for smaller budget non-durable items. Similarly, since duties magnify price changes, a similar reasoning should therefore be applied to the elasticity of imports relative to import duties. As most durable items can be considered luxuries, the income elasticity of demand for these items should, in general, be greater than that of nondurables.

Equation (1) allows the coefficients in the model to vary across each product group. This flexible specification permits the evaluation of a number of restrictions that are usually imposed when an aggregate import demand function is estimated. One of the main assumptions of this framework is that the coefficients of the import demand function can be restricted to some representative aggregate demand function. To test this hypothesis, we employed the test for poolability under the general assumption that $\varepsilon \sim N(0, \Omega)$ (see Baltagi 2005, 55-56). F-tests are also undertaken on price, income and the applied duties coefficients to check if they can be restricted across product groups.

The estimated model is then employed to conduct a simulation regarding the potential effects of tariff harmonisation on import demand and tariff receipts. This simulation assumes that the estimated coefficients presented are likely to remain unchanged, at least in the short term. Various tariff harmonisation levels are considered: (1) $10 \%$; (2) $20 \%$; (3) $30 \%$; (4) $40 \%$; 
and (5) $50 \%$. To account for any potential multiplier effects, income is increased by $\Delta y=\frac{1}{1-c} \Delta d$, where $c$ is the marginal propensity to consumer, under the various tariff harmonisation scenarios. This income effect attempts to account for the impact of higher disposable incomes on the demand for imports.

\section{Data sources and descriptive statistics}

Disaggregated monthly observations on imports at the four-digit SITC level for 91 products between January 2000 and September 2008 are utilised in the study. The dataset represents $35 \%$ of the total consumer goods imported into the island. For each product, the ratio of import values to import quantities is employed as a proxy for the import price of each product. Domestic prices are taken from the retail price index database of the Barbados Statistical Service. All prices are in local currency terms (Barbados' exchange rate is fixed to the US dollar: BDS\$2 = US\$1). Data on the actual applied duties were taken from customs database obtained from the Barbados Statistical Service. Real gross domestic product, obtained from the Central Bank of Barbados, is employed as the proxy for domestic activity.

Figure 2 provides the kernel density graphs (non-parametric estimates of the probability density function) for all the variables used in the study. The density functions for all the variables do not have the shapes expected of normally distributed variables. In the case of product-level variables, real imports, price and applied tariffs follow a tent-shaped distribution. This preliminary analysis is in line with the calculated skewness and kurtosis statistics presented in Table 1. For all of the categories considered the skewness statistic is less than one indicating that the data are skewed to the left. There are particularly long left tails for beverages, breads and biscuits, feeding stuffs for animals and dairy product imports. Only in the case of chemical and related products is the measure of kurtosis close to three, for all the other product categories the relatively large kurtosis statistics suggests that importation of the respective product volume into the island have a peaked distribution.

In terms of the comparison between durable and non-durable goods, the statistics in Table 1 implies that durable goods imports tend to be less volatile than that for non-durables. The coefficient of variation for durable goods imports into the island was 0.131 , compared to 0.216 non-durable goods imports. The finding that the volatility of durable goods is lower than that for non-durables is not surprising, as businesses tend to vary their orders for non-durables in an attempt to reduce losses due to spoilage. The skewness and kurtosis of both series depart significantly from that expected from a series that is normally distributed. The calculated skewness and kurtosis for non-durable goods were -1.267 and 8.874 , respectively, while 

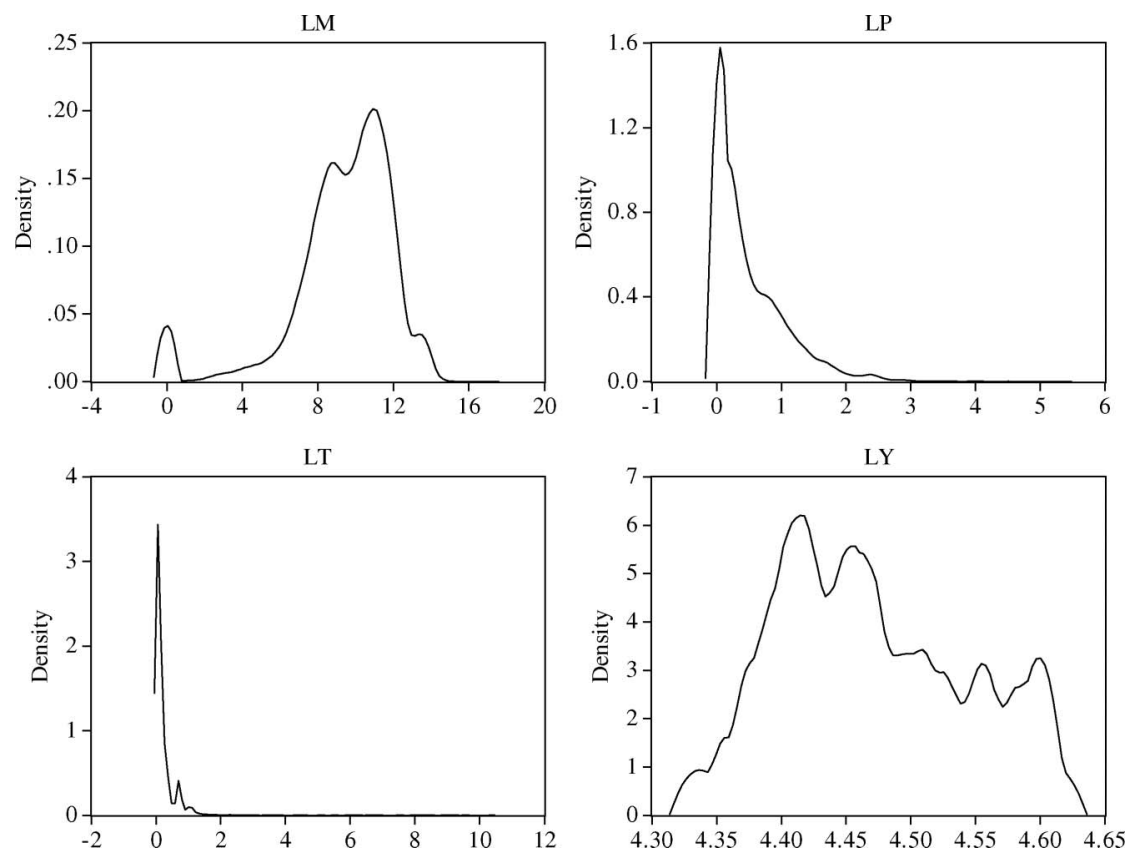

Figure 2. Kernel density graphs for real imports, relative import prices and duties applied.

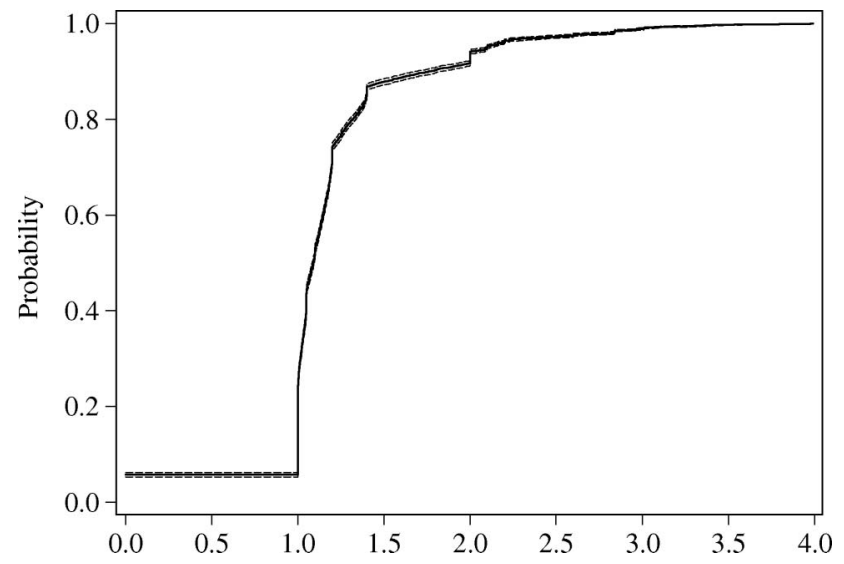

Figure 3. Empirical cumulative distribution of applied tariffs.

that of durable goods were -0.446 and 4.616. The Jarque-Bera test confirmed that both series are non-normal, suggesting that orders for imported goods are subject to infrequent extreme outliers. Figures 3 and 4 


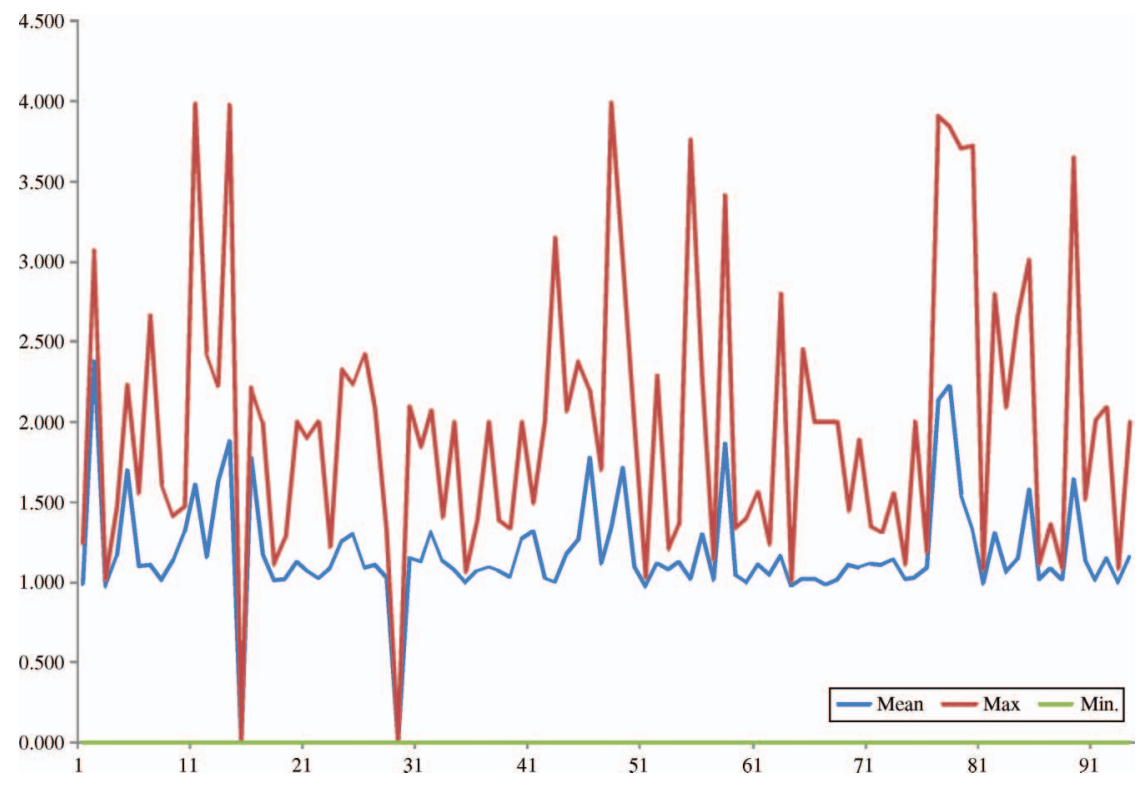

Figure 4. Maximum and minimum applied tariffs $(1+\mathrm{t})$ for various imported product categories.

suggest that most of the applied tarrifs tend to range between $0 \%$ and $60 \%$ over the review period, with the tarrifs on some items rising to above $200 \%$.

To further evaluate the dynamic properties of the series, panel unit root statistics were calculated for the product-level series, real imports, relative prices and duties applied, while the usual time series augmented DickeyFuller (ADF) statistic was applied to income. Four panel unit root statistics (Levin, Lin and Chu t* 2002; Im, Pesaran and Chin w* 2003; ADF Fisher chi-square; and PP Fisher chi-square) were calculated and the results are provided in Table 2. The $p$-values provided in the table in square brackets suggest that the null hypothesis of a unit root in the series employed in this study could not be accepted at normal levels of testing.

\section{Empirical findings}

\subsection{Estimated equation}

The pooled import demand equation results, which are similar to the traditional aggregate demand function that would normally be estimated, are provided in Table 3. In accordance with a priori reasoning, the coefficient on the price and applied tariff variables are negative and statistically significant, while the null hypothesis that the income elasticity can be restricted to zero could not be rejected at normal levels of testing. These results suggest that increases in the prices and tariffs on imported 
Table 1. Descriptive statistics for various import categories.

\begin{tabular}{|c|c|c|c|c|c|c|c|}
\hline & Mean & Max. & Min. & $\begin{array}{c}\text { Coef. } \\
\text { Var. }\end{array}$ & Skew. & Kurt. & Obs. \\
\hline Beverages & 10.772 & 13.061 & 5.788 & 0.107 & -1.312 & 9.363 & 105.000 \\
\hline Aerated beverages & 13.437 & 14.338 & 11.665 & 0.035 & -0.936 & 4.185 & 105.000 \\
\hline Flavoured preparations & 10.588 & 11.746 & 8.108 & 0.059 & -1.053 & 4.886 & 105.000 \\
\hline Flavoured syrups & 8.768 & 12.470 & 6.957 & 0.135 & 1.306 & 4.319 & 105.000 \\
\hline Malted beverages & 11.621 & 14.390 & 0.000 & 0.150 & -4.338 & 26.491 & 105.000 \\
\hline Orange juice & 10.906 & 12.647 & 7.996 & 0.082 & -0.381 & 3.184 & 105.000 \\
\hline Pineapple juice & 9.309 & 12.772 & 0.000 & 0.178 & -2.469 & 13.114 & 105.000 \\
\hline Meats and fish & 9.156 & 11.782 & 4.151 & 0.245 & -1.074 & 7.045 & 105.000 \\
\hline Bacon & 4.852 & 8.804 & 0.000 & 0.564 & -0.577 & 2.229 & 105.000 \\
\hline Beef & 11.661 & 13.351 & 9.824 & 0.044 & -0.219 & 4.601 & 105.000 \\
\hline Corned beef & 10.529 & 12.554 & 0.000 & 0.178 & -3.954 & 21.016 & 105.000 \\
\hline Flying fish & 7.949 & 11.568 & 0.000 & 0.351 & -2.104 & & 105.000 \\
\hline Food preparations & 8.714 & 11.907 & 6.957 & 0.122 & 1.037 & & 105.000 \\
\hline Lunches & 3.848 & 9.786 & 0.000 & 1.026 & 0.243 & & 105.000 \\
\hline Mackerels & 8.940 & 11.678 & 0.000 & 0.382 & -1.821 & 4.8 & 105.000 \\
\hline Pork & 11.276 & 12.545 & 6.849 & 0.067 & -2.162 & 12.7 & 105.000 \\
\hline Salmon & 8.813 & 10.5 & 5.602 & 0.094 & -0.776 & 4.4 & 105.000 \\
\hline Salted pork & 11.125 & 12.369 & 9.816 & 0.041 & 0.079 & 3.1 & 105.000 \\
\hline Sardines & 10.022 & 11.674 & 0.000 & 0.159 & -3.493 & 18.5 & 105.000 \\
\hline Sausages & 7.775 & 9.905 & 3.738 & 0.137 & -0.811 & 4.6 & 105.000 \\
\hline Sheep & 11.646 & 14.711 & 10.316 & 0.051 & 0.926 & & 105.000 \\
\hline Tuna & 11.061 & 12.858 & 9.157 & 0.055 & -0.450 & & 105.000 \\
\hline Turkey wings & 9.134 & 12.445 & 0.000 & 0.400 & -2.023 & 5.3 & 105.000 \\
\hline Fruits and vegetables & 9.565 & 11.693 & 5.402 & 0.198 & -1.003 & 5.3 & 104.526 \\
\hline Bananas & 12.204 & 12.847 & 11.422 & 0.020 & -0.503 & & 105.000 \\
\hline Beets & 6.391 & 8.922 & 0.000 & 0.288 & -1.878 & 6.9 & 105.000 \\
\hline Broccoli & 10.852 & 11.564 & 9.881 & 0.031 & -0.560 & 3.1 & 105.000 \\
\hline Cabbage & 10.425 & 11.823 & 8.103 & 0.050 & -0.866 & & 105.000 \\
\hline Canned corn & 9.158 & 11.100 & 3.135 & 0.129 & -1.694 & 8.984 & 105.000 \\
\hline Carrots & 10.119 & 11.033 & 6.485 & 0.079 & -2.645 & 10.651 & 105.000 \\
\hline Cucur & 5.808 & 10.240 & 0.000 & 0.509 & -0.781 & 2.530 & 105.000 \\
\hline Engli & 11.858 & 12.595 & 10.685 & 0.029 & -0.625 & 4.2 & 105.000 \\
\hline English $\mathrm{p}$ & 13.557 & 14.087 & 13.146 & 0.012 & 0.036 & 3.975 & 96.000 \\
\hline Grapefruits & 9.382 & 10.953 & 0.000 & 0.280 & -2.930 & 10.208 & 105.000 \\
\hline Grapes & 11.093 & 12.272 & 10.338 & 0.028 & 1.098 & & 105.000 \\
\hline Lettu & 9.010 & 11.504 & 6.019 & 0.104 & 0.113 & 3.0 & 105.000 \\
\hline Onions & 11.275 & 12.940 & 7.245 & 0.115 & -1.287 & 4.033 & 105.000 \\
\hline Oran: & 0.375 & 12.498 & 0.000 & 0.379 & -2.255 & 6.141 & 105.000 \\
\hline Plant & 11.279 & 11.998 & 9.702 & 0.044 & -1.566 & 5.337 & 105.000 \\
\hline Pump & 9.056 & 11.351 & 0.000 & 0.276 & -2.372 & 8.427 & 105.000 \\
\hline Sweet & 5.032 & 10.802 & 0.000 & 0.660 & -0.438 & 1.874 & 105.000 \\
\hline Tomatoes & 9.537 & 14.053 & 6.468 & 0.178 & 0.723 & 3.580 & 105.000 \\
\hline Yams & 5.316 & 9.587 & 0.000 & 0.555 & -0.624 & 2.351 & 105.000 \\
\hline Breads and biscuits & 8.518 & 10.381 & 1.523 & 0.218 & -2.469 & 9.230 & 105.000 \\
\hline Sweet biscuits & 10.644 & 11.839 & 3.045 & 0.148 & -3.059 & 11.478 & 105.000 \\
\hline Biscuits (other) & 6.391 & 8.922 & 0.000 & 0.288 & -1.878 & 6.981 & 105.000 \\
\hline
\end{tabular}


Table 1. (Continued).

\begin{tabular}{|c|c|c|c|c|c|c|c|}
\hline & Mean & Max. & Min. & $\begin{array}{l}\text { Coef. } \\
\text { Var. }\end{array}$ & Skew. & Kurt. & Obs. \\
\hline $\begin{array}{l}\text { Condiments, sugars, } \\
\text { spices and teas }\end{array}$ & 9.299 & 12.410 & 5.075 & 0.158 & -0.566 & 13.223 & 104.182 \\
\hline Black pepper & 8.229 & 9.596 & 6.312 & 0.075 & -0.390 & 3.029 & 96.000 \\
\hline Brown sugar & 8.614 & 14.006 & 0.000 & 0.563 & -0.501 & 1.795 & 105.000 \\
\hline Curry powder & 8.343 & 11.752 & 6.435 & 0.124 & 0.230 & 2.772 & 105.000 \\
\hline Green tea & 7.854 & 9.542 & 0.000 & 0.142 & -3.418 & 24.470 & 105.000 \\
\hline Jams & 10.623 & 12.307 & 8.586 & 0.078 & -0.584 & 2.606 & 105.000 \\
\hline Pepper sauce & 7.960 & 12.822 & 5.182 & 0.185 & 1.746 & 6.248 & 105.000 \\
\hline Spices & 8.111 & 10.346 & 6.260 & 0.088 & -0.007 & 3.819 & 105.000 \\
\hline Table salt & 8.832 & 12.470 & 4.407 & 0.187 & 0.010 & 2.450 & 105.000 \\
\hline Tomato ketchup & 11.527 & 12.709 & 6.014 & 0.105 & -2.843 & 10.864 & 105.000 \\
\hline Tomato sauce & 8.974 & 16.863 & 7.633 & 0.105 & 5.448 & 47.178 & 105.000 \\
\hline White sugar & 13.220 & 14.094 & 4.997 & 0.086 & -5.915 & 40.222 & 105.000 \\
\hline $\begin{array}{l}\text { Feeding stuffs for } \\
\quad \text { animals }\end{array}$ & 8.501 & 11.487 & 0.000 & 0.395 & -2.856 & 18.822 & 105.000 \\
\hline Cattle feed & 6.009 & 10.087 & 0.000 & 0.659 & -0.748 & 1.768 & 105.000 \\
\hline Poultry feed & 10.992 & 12.887 & 0.000 & 0.131 & -4.964 & 35.875 & 105.000 \\
\hline Dairy products & 9.538 & 12.672 & 4.097 & 0.217 & -1.546 & 8.549 & 104.571 \\
\hline Cheese & 12.162 & 13.468 & 11.154 & 0.030 & 0.178 & 4.960 & 105.000 \\
\hline Condensed milk & 10.943 & 15.088 & 0.000 & 0.183 & -4.344 & 24.038 & 105.000 \\
\hline Cooking butter & 9.092 & 12.345 & 2.890 & 0.132 & -2.122 & 10.988 & 105.000 \\
\hline Evaporated milk & 6.386 & 12.823 & 0.000 & 0.780 & -0.273 & 1.309 & 102.000 \\
\hline Margarine & 8.427 & 11.622 & 0.000 & 0.226 & -1.945 & 8.852 & 105.000 \\
\hline Mayonnaise & 10.686 & 11.513 & 8.889 & 0.051 & -1.544 & 5.563 & 105.000 \\
\hline Table butter & 9.067 & 11.844 & 5.749 & 0.118 & -0.770 & 4.136 & 105.000 \\
\hline $\begin{array}{c}\text { Cereals and cereal } \\
\text { preparations }\end{array}$ & 9.632 & 12.696 & 3.445 & 0.154 & -1.121 & 6.368 & 105.000 \\
\hline Corn flakes & 9.092 & 12.345 & 2.890 & 0.132 & -2.122 & 10.988 & 105.000 \\
\hline Rice (bulk) & 10.105 & 14.319 & 0.000 & 0.256 & -0.426 & 3.972 & 105.000 \\
\hline Rice (pack) & 10.681 & 13.693 & 4.522 & 0.124 & -1.683 & 8.086 & 105.000 \\
\hline Oats & 8.650 & 10.427 & 6.366 & 0.104 & -0.252 & 2.426 & 105.000 \\
\hline $\begin{array}{c}\text { Animal, vegetable } \\
\text { oils and fats }\end{array}$ & 10.026 & 12.439 & 5.698 & 0.127 & -0.511 & 6.987 & 105.000 \\
\hline Corn oil & 10.377 & 11.581 & 7.143 & 0.084 & -1.744 & 6.443 & 105.000 \\
\hline Natural honey & 9.245 & 14.120 & 7.460 & 0.156 & 2.279 & 7.420 & 105.000 \\
\hline Peanut butter & 9.353 & 10.541 & 8.188 & 0.069 & 0.122 & 1.931 & 105.000 \\
\hline Vegetable oils & 11.128 & 13.513 & 0.000 & 0.198 & -2.700 & 12.154 & 105.000 \\
\hline $\begin{array}{l}\text { Chemical and } \\
\text { related products }\end{array}$ & 8.397 & 12.110 & 4.504 & 0.342 & -0.217 & 3.851 & 103.875 \\
\hline Detergent & 11.774 & 12.954 & 9.734 & 0.054 & -0.823 & 3.382 & 105.000 \\
\hline Herbicides & 9.804 & 14.471 & 5.257 & 0.166 & -0.484 & 3.570 & 105.000 \\
\hline Insecticides & 2.860 & 11.368 & 0.000 & 1.351 & 0.772 & 1.882 & 105.000 \\
\hline Liquid bleach & 11.933 & 13.089 & 10.841 & 0.031 & -0.070 & 3.553 & 105.000 \\
\hline Liquid detergents & 9.276 & 13.083 & 3.219 & 0.167 & -0.417 & 5.069 & 105.000 \\
\hline Mosquito coils & 6.416 & 10.453 & 0.000 & 0.560 & -0.814 & 2.134 & 105.000 \\
\hline & 7.455 & 11.733 & 5.598 & 0.179 & 1.711 & 5.830 & 105.000 \\
\hline Scouring powder & 7.660 & 9.731 & 1.386 & 0.226 & -1.611 & 5.384 & 96.000 \\
\hline
\end{tabular}


Table 1. (Continued).

\begin{tabular}{lrrrrrrr}
\hline & & \multicolumn{7}{c}{ Coef. } \\
& Mean & Max. & Min. & Var. & Skew. & Kurt. & Obs. \\
\hline Durable goods & 8.868 & 11.312 & 5.591 & 0.131 & -0.446 & 4.616 & 103.615 \\
Blenders & 7.972 & 9.574 & 6.333 & 0.097 & -0.159 & 2.343 & 105.000 \\
Brooms & 5.939 & 8.868 & 0.000 & 0.283 & -1.058 & 5.030 & 105.000 \\
Colour television & 10.999 & 13.370 & 9.807 & 0.049 & 0.616 & 5.795 & 96.000 \\
Deep freezers & 8.675 & 11.773 & 4.277 & 0.220 & -0.277 & 2.129 & 105.000 \\
Electronic irons & 8.489 & 9.965 & 6.444 & 0.086 & -0.364 & 2.768 & 105.000 \\
Electronic stoves & 8.489 & 9.965 & 6.444 & 0.086 & -0.364 & 2.768 & 105.000 \\
Gas stoves & 10.504 & 11.621 & 8.802 & 0.060 & -0.508 & 2.904 & 96.000 \\
Microwave & 10.686 & 11.513 & 8.889 & 0.051 & -1.544 & 5.563 & 105.000 \\
Portable radios & 7.973 & 13.329 & 0.000 & 0.286 & -1.640 & 6.936 & 105.000 \\
Refrigerators & 8.508 & 11.702 & 6.748 & 0.112 & 0.640 & 3.620 & 105.000 \\
Room fans & 9.782 & 13.884 & 7.915 & 0.079 & 1.027 & 9.558 & 105.000 \\
Vacuum cleaners & 8.536 & 9.623 & 7.018 & 0.060 & -0.447 & 3.287 & 105.000 \\
Washing machines & 8.731 & 11.873 & 0.000 & 0.240 & -1.715 & 7.310 & 105.000 \\
\hline
\end{tabular}

Table 2. Panel unit root statistics for imported goods.

\begin{tabular}{lcccc}
\hline $\begin{array}{c}\text { Levin, Lin } \\
\text { \& Chu t* }\end{array}$ & $\begin{array}{c}\text { Im, Pesaran } \\
\text { and Shin W-stat }\end{array}$ & $\begin{array}{c}\text { ADF-Fisher } \\
\text { chi-square }\end{array}$ & $\begin{array}{c}\text { PP-Fisher } \\
\text { chi-square }\end{array}$ \\
\hline$l m$ & $-35.593[0.000]$ & $-45.024[0.000]$ & $2555.72[0.000]$ & $3813.73[0.000]$ \\
$l p$ & $-38.127[0.000]$ & $-45.100[0.000]$ & $2393.37[0.000]$ & $2518.31[0.000]$ \\
$l d$ & $-122.756[0.000]$ & $-77.001[0.000]$ & $3414.70[0.000]$ & $3624.78[0.000]$ \\
\hline
\end{tabular}

Notes: (1) Income had an ADF test statistic of -3.971 which is significant at the $5 \%$ level of testing. (2) $P$-values provided in square brackets below coefficients.

Table 3. Pooled import demand results.

\begin{tabular}{lcr}
\hline $\begin{array}{l}\text { Dependent variable: } \\
\text { Real import demand }\end{array}$ & Pooled OLS & \multicolumn{1}{c}{ Fixed effects } \\
\hline$c$ & $9.547(1.669)^{* * * *}$ & $4.597(1.402)^{* * *}$ \\
$l p$ & $-0.309(0.048)^{* * *}$ & $-0.372(0.069)^{* * *}$ \\
$l t$ & $-1.386(0.052)^{* * *}$ & $-0.789(0.132)^{* * *}$ \\
$l y$ & $0.063(0.374)$ & $1.203(0.314)^{* * *}$ \\
r-squared & 0.072 & 0.580 \\
s.e. regression & 2.615 & 1.760 \\
Obs & 9482 & 9482 \\
\hline
\end{tabular}

Notes: (1) White robust standard errors are provided in parentheses below coefficients. (2) $* * *$ indicates significance at the $1 \%$ level of testing.

goods leads to a reduction in demand for these items. The coefficient on the relative price variable indicates that the demand for imports in Barbados is relatively price inelastic, i.e. demand is not very responsive to changes in income. 
Table 3 provides the results from relaxing the assumption that the equation intercept is the same across all product categories. An F-test was conducted to evaluate whether or not the regression fixed effects could be restricted to zero. The calculated F-statistic was 126.146[0.000], suggesting that the null hypothesis that the fixed effects are not significantly different from zero should be rejected at the levels of testing. Only the fixed effects coefficient estimates are presented in Table 3 as the Hausman test for correlated random effects had a chi-square statistic of 12.371[0.006], indicating that disturbances may be correlated with the explanatory variables making the random effects estimator biased and inconsistent.

The coefficient estimates obtained from the fixed effects specification differ somewhat when compared to the pooled equation results. The elasticity estimate for applied duties remains negative and significant; however, it is now less than one suggesting a less than proportionate response of imports to tariff rate changes. Another change in the estimated equation results is that the income elasticity is now statistically different from zero, with a coefficient greater than zero. This result indicates that a $1 \%$ increase in income is likely to lead to a more than $1 \%$ rise in imports, and consequently the import to GDP ratio should climb over time. This is indeed the case, as the import to GDP ratio rose from $57 \%$ in 2000 to $61 \%$ in 2008 .

Both the pooled and fixed effects equation results assume that the slope coefficients are the same across each product category. However, given the wide variety of goods imported into most countries this assumption is somewhat heroic. Table 4 provides F-tests for the null hypothesis that the coefficients on relative prices, tariffs and income can be restricted across products. The results provided in the table suggest that the null hypothesis cannot be accepted at the normal levels of testing for all the variables included in the regression model. These results therefore suggest that a disaggregate import demand model appears to be the most appropriate specification.

Major product-level groupings are provided for ease of analysis. In relation to beverages, these products tended to be inelastic in response to price changes (elasticity less than one). Similar findings are obtained in relation to meats, fruits and vegetables, breads and biscuits, condiments,

Table 4. Test for cross-section specific coefficients.

\begin{tabular}{lr}
\hline & \multicolumn{1}{c}{ F-statistic } \\
\hline$l p$ & $87.292[0.000]$ \\
$l t$ & $1375.598[0.000]$ \\
$l y$ & $377.607[0.000]$ \\
\hline
\end{tabular}

Note: $P$-values provided in square brackets below coefficients. 
Table 5. Results for individual-specific coefficient estimates.

\begin{tabular}{|c|c|c|c|}
\hline & $l p$ & ly & lt \\
\hline \multicolumn{4}{|l|}{ Beverages } \\
\hline Aerated beverages & $-0.237(0.111)^{*}$ & $0.414(0.618)$ & $1.373(2.034)$ \\
\hline Flavoured preparations & $0.129(0.090)^{*}$ & $-1.811(1.030)^{*}$ & $3.152(4.280)$ \\
\hline Flavoured syrups & $-0.754(0.180)^{*}$ & $9.928(1.214)^{*}$ & $-8.852(1.406)^{*}$ \\
\hline Malted beverages & $-0.764(0.894)$ & $1.699(2.717)$ & $-5.768(16.483)$ \\
\hline Orange juice & $-0.573(0.151)^{*}$ & $-3.605(0.928)^{*}$ & $-7.117(1.838)^{*}$ \\
\hline Pineapple juice & $-0.566(0.356)^{*}$ & $-3.552(1.771)^{*}$ & $-4.484(1.373)^{*}$ \\
\hline \multicolumn{4}{|l|}{ Meats and fish } \\
\hline Bacon & $0.766(0.378)^{*}$ & $14.011(3.537)^{*}$ & $1.891(0.673)^{*}$ \\
\hline Beef & $-1.069(0.513)^{*}$ & $2.584(0.935)^{*}$ & $-0.586(0.747)$ \\
\hline Corned beef & $-0.778(0.636)^{*}$ & $-1.659(2.023)$ & $-14.205(3.350)^{*}$ \\
\hline Flying fish & $-0.537(0.797)$ & $-4.228(1.875)^{*}$ & $-9.323(1.728)^{*}$ \\
\hline Food preparations & $-0.587(0.094)^{*}$ & $9.369(0.909)^{*}$ & $-6.597(1.128)^{*}$ \\
\hline Luncheon meats & $2.382(0.730)^{*}$ & $-26.171(3.318)^{*}$ & $0.51(0.171)^{*}$ \\
\hline Mackerels & $-0.993(0.538)^{*}$ & $-9.941(4.865)^{*}$ & $-0.997(1.213)$ \\
\hline Pork & $0.147(0.955)$ & $1.486(1.227)$ & $0.022(0.039)$ \\
\hline Salmon & $-0.029(0.124)$ & $3.748(1.195)^{*}$ & $3.216(1.347)^{*}$ \\
\hline Salted pork & $-1.1(0.356)^{*}$ & $1.976(0.749)^{*}$ & $2.515(3.332)$ \\
\hline Sardines & $-0.137(0.215)$ & $-2.221(1.509)$ & $-14.57(1.729)^{*}$ \\
\hline Sausages & $0.221(0.335)$ & $2.248(1.348)^{*}$ & $-0.097(0.097)$ \\
\hline Sheep & $-1.129(0.757)^{*}$ & $1.720(1.002)^{*}$ & $0.01(0.163)$ \\
\hline Tuna & $-0.558(0.176)^{*}$ & $2.849(0.923)^{*}$ & $1.735(4.603)$ \\
\hline Turkey wings & $-1.867(2.697)$ & $-20.767(4.464)^{*}$ & $-2.907(1.201)^{*}$ \\
\hline \multicolumn{4}{|l|}{ Fruits and vegetables } \\
\hline Bananas & $-0.021(0.383)$ & $0.095(0.448)$ & $3.7(20.765)$ \\
\hline Beets & $0.133(1.098)$ & $-2.343(4.116)$ & $-2.037(1.335)^{*}$ \\
\hline Broccoli & $-0.122(0.074)$ & $3.475(0.380)^{*}$ & $0.958(0.391)^{*}$ \\
\hline Cabbage & $-2.122(0.592)^{*}$ & $2.234(0.836)^{*}$ & $-0.774(0.333)^{*}$ \\
\hline Canned corn & $-0.137(0.303)$ & $4.337(1.225)^{*}$ & $-0.858(0.286)^{*}$ \\
\hline Carrots & $0.113(0.568)$ & $1.295(1.242)$ & $2.654(0.622)^{*}$ \\
\hline Cucumbers & $-0.039(-0.561)$ & $-8.61(2.130)^{*}$ & $-7.069(0.673)^{*}$ \\
\hline English apples & $-1.064(0.362)^{*}$ & $0.63(0.556)^{*}$ & $-0.326(0.271)^{*}$ \\
\hline English potatoes & $0.041(0.166)$ & $0.317(0.357)$ & $1.84(0.602)^{*}$ \\
\hline Grapefruits & $-9.578(1.854)^{*}$ & $-5.549(2.507)^{*}$ & $14.700(4.082)$ \\
\hline Grapes & $-0.65(0.153)^{*}$ & $0.282(0.394)$ & $-1.485(0.354)^{*}$ \\
\hline Lettuce & $-1.747(0.249)^{*}$ & $-0.081(1.325)$ & $-0.988(0.337)^{*}$ \\
\hline Onions & $-6.06(0.561)^{*}$ & $0.356(1.200)$ & $-0.696(0.251)^{*}$ \\
\hline Oranges & $1.220(2.412)$ & $-7.463(3.852)^{*}$ & $11.346(6.220)^{*}$ \\
\hline Plantains & $-3.241(0.978)^{*}$ & $-1.075(0.854)$ & $26.772(-27.393)$ \\
\hline Pumpkins & $-0.93(0.289)^{*}$ & $0.058(3.361)$ & $-13.398(0.773)^{*}$ \\
\hline Sweet potatoes & $2.758(1.501)^{*}$ & $-9.495(3.863)^{*}$ & $-5.107(1.065)^{*}$ \\
\hline Tomatoes & $-1.662(0.212)^{*}$ & 4.209 (1.767)* & $-2.996(0.469) *$ \\
\hline Yams & $0.567(0.546)$ & $-10.516(2.392)^{*}$ & $-8.502(0.516)^{*}$ \\
\hline \multicolumn{4}{|l|}{ Breads and biscuits } \\
\hline Sweet biscuits & $0.077(0.138)$ & $-11.115(2.270)^{*}$ & $6.244(1.932)^{*}$ \\
\hline Biscuits (other) & $0.041(0.076)$ & $3.707(0.698)^{*}$ & $-2.428(1.349)$ \\
\hline
\end{tabular}


Table 5. (Continued).

\begin{tabular}{|c|c|c|c|}
\hline & $l p$ & $l y$ & $l t$ \\
\hline \multicolumn{4}{|c|}{ Condiments, sugars, spices and teas } \\
\hline Black pepper & $-0.203(0.116)$ & $0.611(0.988)$ & $-2.781(0.838)^{*}$ \\
\hline Brown sugar & $-1.952(0.276)^{*}$ & $1.063(3.671)$ & $-14.151(1.952)^{*}$ \\
\hline Curry powder & $-0.356(0.137)^{*}$ & $0.851(1.314)$ & $1.134(0.751) *$ \\
\hline Green tea & $-0.443(0.152) *$ & $3.815(1.192)^{*}$ & $-9.215(2.834)$ \\
\hline Jams & $-0.124(0.113)$ & $1.575(0.741)^{*}$ & $-3.212(0.295)^{*}$ \\
\hline Pepper sauce & $-1.472(0.166)^{*}$ & $13.224(1.299)^{*}$ & $-7.519(1.785)^{*}$ \\
\hline Spices & $-0.567(0.088)^{*}$ & $1.623(0.974)^{*}$ & $-4.406(4.739)$ \\
\hline Table salt & $-1.219(0.268)^{*}$ & $5.959(2.009)^{*}$ & $-3.132(2.143)$ \\
\hline Tomato ketchup & $-1.912(0.253)^{*}$ & $-2.567(1.068)^{*}$ & $11.161(3.889)^{*}$ \\
\hline Tomato sauce & $-0.109(0.320)$ & $2.99(1.542)^{*}$ & $2.562(1.233)^{*}$ \\
\hline White sugar & $-6.234(2.008)^{*}$ & $2.853(1.159)^{*}$ & $17.765(5.096)^{*}$ \\
\hline \multicolumn{4}{|c|}{ Feeding stuffs for animals } \\
\hline Cattle feed & $1.458(1.168)$ & $-3.343(2.675)$ & $-10.177(0.754)$ \\
\hline Poultry feed & $0.770(0.665)$ & $3.043(1.547)^{*}$ & $-15.663(0.287)^{*}$ \\
\hline \multicolumn{4}{|l|}{ Dairy products } \\
\hline Cheese & $-0.154(0.066)^{*}$ & $1.118(0.605)$ & $0.130(2.370)$ \\
\hline Condensed milk & $-0.834(1.098)$ & $-1.58(2.573)$ & $-8.776(4.343)^{*}$ \\
\hline Cooking butter & $-0.776(0.213)^{*}$ & $2.53(1.525)^{*}$ & $-0.083(0.203)$ \\
\hline Evaporated milk & $5.596(0.989)^{*}$ & $-1.172(3.828)$ & $-7.828(1.291)^{*}$ \\
\hline Margarine & $-1.205(0.315)^{*}$ & $6.136(1.647)^{*}$ & $-6.549(1.293)^{*}$ \\
\hline Mayonnaise & $-0.001(0.111)$ & $-0.666(0.765)$ & $5.667(1.570)^{*}$ \\
\hline Table butter & $-0.683(0.295)^{*}$ & $3.044(1.441)^{*}$ & $-2.384(1.772)$ \\
\hline \multicolumn{4}{|c|}{ Cereals and cereal preparations } \\
\hline Corn flakes & $-1.392(0.390)^{*}$ & $3.046(3.070)$ & $-6.547(3.496)^{*}$ \\
\hline Rice (bulk) & $-1.362(0.314)^{*}$ & $-1.163(2.213)$ & $-1.883(0.288)^{*}$ \\
\hline Rice (pack) & $-0.772(0.810)$ & $9.04(1.458)^{*}$ & $-1.444(1.671)$ \\
\hline Oats & $-0.102(0.262)$ & $8.014(1.233)^{*}$ & $0.762(4.195)$ \\
\hline \multicolumn{4}{|c|}{ Animal, vegetable oils and fats } \\
\hline Corn oil & $0.366(0.285)^{*}$ & $-2.155(1.370)^{*}$ & $2.648(1.162)^{*}$ \\
\hline Natural honey & $-0.521(0.202)^{*}$ & $6.02(1.719)^{*}$ & $-6.206(1.763)^{*}$ \\
\hline Peanut butter & $-0.106(0.147)$ & $1.507(0.894)^{*}$ & $3.568(0.931) *$ \\
\hline Vegetable oils & $-1.7(0.339)^{*}$ & $2.249(1.763)$ & $-16.401(1.289)^{*}$ \\
\hline \multicolumn{4}{|c|}{ Chemical and related products } \\
\hline Detergent & $-1.921(0.646)^{*}$ & $1.291(0.797)^{*}$ & $-7.963(1.275)^{*}$ \\
\hline Herbicides & $0.549(0.498)$ & $2.286(2.195)$ & $-3.633(0.314)^{*}$ \\
\hline Insecticides & $-0.267(0.529)$ & $35.179(2.830)^{*}$ & $-0.689(0.258)^{*}$ \\
\hline Liquid bleach & $0.035(0.061)$ & $1.358(0.557)^{*}$ & $-0.392(0.459)$ \\
\hline Liquid detergents & $-0.472(0.200)^{*}$ & $7.379(1.220)^{*}$ & $-2.021(0.195)^{*}$ \\
\hline Mosquito coils & $-0.289(-0.401)$ & $3.861(-4.375)$ & $-1.037(0.128)^{*}$ \\
\hline Scouring pads & $-0.081(0.146)$ & $8.217(1.394)^{*}$ & $-1.200(0.195)^{*}$ \\
\hline Scouring powder & $-0.121(0.451)$ & $3.051(1.601)^{*}$ & $-1.629(0.164) *$ \\
\hline \multicolumn{4}{|l|}{ Durable goods } \\
\hline Blenders & $-0.266(0.158)$ & $5.739(0.940)^{*}$ & $0.167(1.150)$ \\
\hline Brooms & $-0.156(0.322)$ & $4.229(1.181)^{*}$ & $-0.923(0.110)^{*}$ \\
\hline Colour TVs & 8.625 (7.948) & $2.567(0.674)^{*}$ & $1.251(1.448)$ \\
\hline
\end{tabular}


Table 5. (Continued).

\begin{tabular}{lccr}
\hline & $l p$ & $l y$ & $l t$ \\
\hline Deep freezers & $-14.388(3.378)^{*}$ & $20.376(1.546)^{*}$ & $-0.069(0.637)$ \\
Electronic irons & $-0.675(0.683)$ & $1.301(0.679)^{*}$ & $1.532(0.555)$ \\
Electronic stoves & $15.326(8.833)^{*}$ & $3.073(1.080)^{*}$ & $1.392(1.066)^{*}$ \\
Gas stoves & $12.638(-15.971)$ & $0.05(-0.822)$ & $3.277(1.515)^{*}$ \\
Microwave & $-0.027(-1.861)$ & $0.46(-0.676)$ & $4.583(1.186)^{*}$ \\
Portable radios & $23.793(4.667)^{*}$ & $3.437(2.580)^{*}$ & $-11.326(0.708)^{*}$ \\
Refrigerators & $1.257(0.408)^{*}$ & $3.861(1.181)^{*}$ & $-3.741(1.485)^{*}$ \\
Room fans & $0.084(0.176)$ & $3.318(0.834)^{*}$ & $5.67(1.164)^{*}$ \\
Vacuum cleaners & $2.053(1.284)^{*}$ & $1.821(0.789)^{*}$ & $2.109(0.885)^{*}$ \\
Washing machines & $-0.397(1.049)$ & $12.165(2.049)^{*}$ & $-2.798(3.593)$ \\
\hline
\end{tabular}

Notes: (1) White robust standard errors are provided in parentheses below coefficients. (2) *indicates significance at the $10 \%$ levels of testing. (3) Seasonal dummies included in regressions.

Table 6. Forecast evaluation statistics for model.

\begin{tabular}{lccc}
\hline & RMSE & MAPE & Thiel \\
\hline Disaggregated model & 2.224 & 14.249 & 0.112 \\
Pooled model & 2.700 & 19.786 & 0.139 \\
Morgan-Granger-Newbold (1977) Test & $-0.337(0.028)$ & & \\
\hline
\end{tabular}

sugars, spices and teas, dairy products and animal vegetable oils and fats. In contrast, cereals and cereal preparations as well as durable goods were more responsive to price changes. As it relates to cereals and cereal preparations, the price elasticity of demand coefficient exceeded one for all of the statistically significant price elasticity coefficients in this category. On a more general level, for durable goods the absolute value of the price elasticity tended to exceed one in most instances.

\subsection{Tariff harmonisation}

The disaggregated import demand equation estimated in Section 4.1 can be used for a number of policy simulations. In this study, the model is employed to assess the possibility of converging tariff rates to some single rate across product categories. Such a change would greatly simplify import procedures for businesses, reduce the administrative burden for government and encourage domestic competition.

Before this simulation is conducted, however, the forecasting properties of the disaggregated equation are first assessed by comparing the forecasts generated to those from the pooled equation. Both equations are estimated over a restricted sample of $2000 \mathrm{M} 1$ to $2006 \mathrm{M} 12$. The equations are then 
Table 7. Simulated effects of tariff harmonisation on import demand (deviation from baseline value).

\begin{tabular}{|c|c|c|c|c|c|}
\hline Import category & $\begin{array}{c}\mathrm{S} 1 \\
(t=10 \%)\end{array}$ & $\begin{array}{c}\mathrm{S} 2 \\
(t=20 \%)\end{array}$ & $\begin{array}{c}\mathrm{S} 3 \\
(t=30 \%)\end{array}$ & $\begin{array}{c}\mathrm{S} 4 \\
(t=40 \%)\end{array}$ & $\begin{array}{c}\mathrm{S} 5 \\
(t=50 \%)\end{array}$ \\
\hline Aerated beverages & 1.022 & 1.007 & 0.993 & 0.981 & 0.969 \\
\hline Bacon & 2.719 & 1.565 & 0.504 & -0.478 & -1.393 \\
\hline Bananas & 0.922 & 0.974 & 1.022 & 1.067 & 1.108 \\
\hline Beef & 1.114 & 1.037 & 0.967 & 0.902 & 0.841 \\
\hline Beets & 0.959 & 0.986 & 1.011 & 1.034 & 1.055 \\
\hline Black pepper & 0.493 & 0.833 & 1.145 & 1.434 & 1.703 \\
\hline Blenders & 1.188 & 1.061 & 0.945 & 0.838 & 0.737 \\
\hline Broccoli & 1.089 & 1.029 & 0.973 & 0.922 & 0.874 \\
\hline Brooms & 1.289 & 1.095 & 0.916 & 0.750 & 0.596 \\
\hline Brown sugar & 1.117 & 1.038 & 0.966 & 0.899 & 0.837 \\
\hline Cabbage & 1.302 & 1.099 & 0.912 & 0.739 & 0.578 \\
\hline Canned corn & 1.238 & 1.078 & 0.931 & 0.795 & 0.668 \\
\hline Canned sausages & 1.091 & 1.029 & 0.973 & 0.921 & 0.872 \\
\hline Carrots & 1.204 & 1.067 & 0.940 & 0.823 & 0.714 \\
\hline Cattle feed & 0.998 & 0.998 & 0.998 & 0.998 & 0.998 \\
\hline Cheese & 1.036 & 1.011 & 0.989 & 0.968 & 0.948 \\
\hline Colour television & 0.933 & 0.977 & 1.018 & 1.055 & 1.090 \\
\hline Condensed milk & 1.034 & 1.011 & 0.990 & 0.970 & 0.952 \\
\hline Cooking butter & 1.043 & 1.014 & 0.987 & 0.962 & 0.939 \\
\hline Corn oil & 1.079 & 1.026 & 0.977 & 0.931 & 0.889 \\
\hline Corned beef & 1.143 & 1.047 & 0.958 & 0.876 & 0.800 \\
\hline Cornflakes & 1.175 & 1.057 & 0.949 & 0.848 & 0.755 \\
\hline Cucumber & 1.321 & 1.105 & 0.907 & 0.723 & 0.552 \\
\hline Curry powder & 0.862 & 0.954 & 1.039 & 1.118 & 1.191 \\
\hline Deep freezers & 0.572 & 0.858 & 1.122 & 1.365 & 1.592 \\
\hline Detergent & 1.060 & 1.019 & 0.982 & 0.947 & 0.915 \\
\hline Disinfectant & 3.020 & 1.664 & 0.418 & -0.737 & -1.811 \\
\hline Electric irons & 1.161 & 1.053 & 0.953 & 0.860 & 0.774 \\
\hline Electric stoves & 0.998 & 0.998 & 0.998 & 0.998 & 0.998 \\
\hline English apples & 1.032 & 1.010 & 0.990 & 0.971 & 0.953 \\
\hline English potatoes & 1.106 & 1.035 & 0.969 & 0.908 & 0.852 \\
\hline Evaporated milk & 1.028 & 1.009 & 0.991 & 0.975 & 0.960 \\
\hline Flavoured preparations & 0.994 & 0.998 & 1.001 & 1.004 & 1.007 \\
\hline Flavoured syrups & 1.037 & 1.011 & 0.988 & 0.967 & 0.946 \\
\hline Flying fish & 0.960 & 0.986 & 1.011 & 1.033 & 1.054 \\
\hline Food preparations & 1.640 & 1.210 & 0.815 & 0.449 & 0.109 \\
\hline Gas stoves & 0.959 & 0.986 & 1.010 & 1.033 & 1.054 \\
\hline Grapefruits & 1.548 & 1.180 & 0.842 & 0.528 & 0.237 \\
\hline Grapes & 0.963 & 0.988 & 1.010 & 1.031 & 1.050 \\
\hline Green tea & 0.265 & 0.757 & 1.210 & 1.629 & 2.020 \\
\hline Herbicides & 1.031 & 1.010 & 0.990 & 0.972 & 0.955 \\
\hline Insecticides & 1.471 & 1.154 & 0.863 & 0.594 & 0.343 \\
\hline Jams & 1.152 & 1.050 & 0.955 & 0.868 & 0.787 \\
\hline Lettuce & 5.711 & 2.551 & -0.356 & -3.048 & -5.553 \\
\hline Liquid bleach & 1.116 & 1.038 & 0.966 & 0.899 & 0.838 \\
\hline
\end{tabular}


Table 7. (Continued).

\begin{tabular}{|c|c|c|c|c|c|}
\hline Import category & $\begin{array}{c}\mathrm{S} 1 \\
(t=10 \%)\end{array}$ & $\begin{array}{c}\mathrm{S} 2 \\
(t=20 \%)\end{array}$ & $\begin{array}{c}\mathrm{S} 3 \\
(t=30 \%)\end{array}$ & $\begin{array}{c}\mathrm{S} 4 \\
(t=40 \%)\end{array}$ & $\begin{array}{c}\mathrm{S} 5 \\
(t=50 \%)\end{array}$ \\
\hline Liquid detergent & 1.005 & 1.001 & 0.998 & 0.995 & 0.992 \\
\hline Luncheon meat & 1.054 & 1.017 & 0.984 & 0.953 & 0.924 \\
\hline Mackerels & 1.362 & 1.119 & 0.895 & 0.688 & 0.495 \\
\hline Malted beverages & -2.215 & -0.061 & 1.922 & 3.757 & 5.465 \\
\hline Margarine & 0.571 & 0.858 & 1.122 & 1.367 & 1.595 \\
\hline Mayonnaise & 1.068 & 1.022 & 0.980 & 0.941 & 0.904 \\
\hline Microwaves & 1.402 & 1.132 & 0.883 & 0.653 & 0.439 \\
\hline Mosquito coils & 0.926 & 0.975 & 1.021 & 1.063 & 1.102 \\
\hline Natural honey & 0.953 & 0.984 & 1.013 & 1.039 & 1.063 \\
\hline Oats & 1.228 & 1.075 & 0.933 & 0.803 & 0.681 \\
\hline Onions & 1.395 & 1.130 & 0.886 & 0.659 & 0.449 \\
\hline Orange juice & 1.446 & 1.147 & 0.871 & 0.616 & 0.378 \\
\hline Oranges & 1.007 & 1.002 & 0.997 & 0.993 & 0.989 \\
\hline Other biscuits & 0.968 & 0.989 & 1.008 & 1.026 & 1.043 \\
\hline Peanut butter & 0.654 & 0.886 & 1.099 & 1.296 & 1.480 \\
\hline Pepper sauce & 1.004 & 1.001 & 0.998 & 0.996 & 0.993 \\
\hline Pineapple juice & 1.868 & 1.286 & 0.749 & 0.253 & -0.209 \\
\hline Plantains & 0.892 & 0.964 & 1.030 & 1.092 & 1.149 \\
\hline Pork & 0.781 & 0.928 & 1.063 & 1.187 & 1.303 \\
\hline Portable radios & 1.082 & 1.027 & 0.976 & 0.929 & 0.885 \\
\hline Poultry feed & 1.122 & 1.040 & 0.964 & 0.894 & 0.829 \\
\hline Pumpkins & 1.587 & 1.193 & 0.830 & 0.494 & 0.182 \\
\hline Refrigerators & 1.153 & 1.050 & 0.955 & 0.867 & 0.785 \\
\hline Rice (bulk) & 1.239 & 1.078 & 0.931 & 0.794 & 0.667 \\
\hline Rice (pack) & 0.932 & 0.977 & 1.019 & 1.057 & 1.093 \\
\hline Room fans & 1.405 & 1.133 & 0.883 & 0.651 & 0.436 \\
\hline Salmon & 1.067 & 1.022 & 0.980 & 0.942 & 0.906 \\
\hline Salted pork & 1.144 & 1.047 & 0.958 & 0.875 & 0.798 \\
\hline Sardines & 1.046 & 1.015 & 0.986 & 0.959 & 0.934 \\
\hline Sauce (other) & 1.124 & 1.040 & 0.963 & 0.891 & 0.825 \\
\hline Sausages & 0.998 & 0.998 & 0.998 & 0.998 & 0.998 \\
\hline Scouring pads & 1.170 & 1.055 & 0.950 & 0.853 & 0.762 \\
\hline Scouring powder & 1.432 & 1.142 & 0.875 & 0.628 & 0.398 \\
\hline Sheep & 1.161 & 1.052 & 0.953 & 0.861 & 0.775 \\
\hline Spices & 1.074 & 1.024 & 0.978 & 0.935 & 0.895 \\
\hline Sweet biscuit & 1.305 & 1.100 & 0.911 & 0.736 & 0.573 \\
\hline Sweet potatoes & 0.591 & 0.865 & 1.116 & 1.349 & 1.566 \\
\hline Table butter & 1.199 & 1.065 & 0.942 & 0.828 & 0.721 \\
\hline Table salt & 1.374 & 1.123 & 0.892 & 0.678 & 0.479 \\
\hline Tomato ketchup & 1.222 & 1.073 & 0.935 & 0.808 & 0.690 \\
\hline Tomato sauce & 0.839 & 0.947 & 1.046 & 1.137 & 1.223 \\
\hline Tomatoes & 1.115 & 1.038 & 0.966 & 0.900 & 0.838 \\
\hline Tuna & 1.090 & 1.029 & 0.974 & 0.922 & 0.874 \\
\hline Turkey wings & 0.131 & 0.713 & 1.249 & 1.745 & 2.207 \\
\hline Vacuum cleaners & 1.042 & 1.013 & 0.987 & 0.963 & 0.940 \\
\hline Vegetable oils & 1.405 & 1.133 & 0.882 & 0.651 & 0.435 \\
\hline
\end{tabular}


Table 7. (Continued).

\begin{tabular}{lccccc}
\hline Import category & $\begin{array}{c}\mathrm{S} 1 \\
(t=10 \%)\end{array}$ & $\begin{array}{c}\mathrm{S} 2 \\
(t=20 \%)\end{array}$ & $\begin{array}{c}\mathrm{S} 3 \\
(t=30 \%)\end{array}$ & $\begin{array}{c}\mathrm{S} 4 \\
(t=40 \%)\end{array}$ & $\begin{array}{c}\mathrm{S} 5 \\
(t=50 \%)\end{array}$ \\
\hline Washing machines & 1.650 & 1.214 & 0.812 & 0.441 & 0.095 \\
White sugar & 0.987 & 0.996 & 1.003 & 1.010 & 1.017 \\
Yams & 0.999 & 0.999 & 0.999 & 0.999 & 0.999 \\
Mean & 1.099 & 1.032 & 0.971 & 0.914 & 0.861 \\
\hline
\end{tabular}

Table 8. Simulated effects of tariff harmonisation on tariff revenue (deviation from baseline value).

\begin{tabular}{lcccrr}
\hline Import category & S1 & S2 & S3 & \multicolumn{2}{c}{$\mathrm{S} 4$} \\
$(t=10 \%)$ & $(t=20 \%)$ & $\begin{array}{c}\text { S5 } \\
(t=30 \%)\end{array}$ \\
$(t=40 \%)$ & $(t=50 \%)$ \\
\hline Aerated beverages & 0.957 & 0.998 & 1.017 & 1.027 & 1.032 \\
Bacon & 3.593 & 1.928 & 0.281 & -1.287 & -2.769 \\
Bananas & 0.851 & 0.962 & 1.047 & 1.119 & 1.182 \\
Beef & 1.050 & 1.031 & 0.991 & 0.945 & 0.898 \\
Beets & 0.792 & 0.960 & 1.070 & 1.155 & 1.226 \\
Black pepper & 0.325 & 0.794 & 1.200 & 1.566 & 1.902 \\
Blenders & 1.127 & 1.059 & 0.970 & 0.877 & 0.785 \\
Broccoli & 0.986 & 1.018 & 1.012 & 0.993 & 0.969 \\
Brooms & 1.232 & 1.099 & 0.942 & 0.782 & 0.627 \\
Brown sugar & 1.049 & 1.032 & 0.992 & 0.945 & 0.897 \\
Cabbage & 1.231 & 1.106 & 0.944 & 0.777 & 0.612 \\
Canned corn & 1.169 & 1.078 & 0.959 & 0.836 & 0.714 \\
Canned sausages & 1.011 & 1.021 & 1.003 & 0.976 & 0.945 \\
Carrots & 1.141 & 1.065 & 0.966 & 0.863 & 0.762 \\
Cattle feed & 0.140 & 0.874 & 1.303 & 1.607 & 1.843 \\
Cheese & 0.937 & 0.999 & 1.025 & 1.038 & 1.044 \\
Colour television & 0.710 & 0.941 & 1.096 & 1.218 & 1.321 \\
Condensed milk & 0.965 & 1.002 & 1.015 & 1.019 & 1.018 \\
Cooking butter & 0.994 & 1.008 & 1.005 & 0.997 & 0.986 \\
Corn oil & 1.005 & 1.018 & 1.004 & 0.982 & 0.957 \\
Corned beef & 1.070 & 1.041 & 0.986 & 0.925 & 0.862 \\
Cornflakes & 1.094 & 1.053 & 0.980 & 0.900 & 0.819 \\
Cucumber & 1.277 & 1.111 & 0.927 & 0.745 & 0.570 \\
Curry powder & 0.748 & 0.934 & 1.078 & 1.202 & 1.313 \\
Deep freezers & 0.248 & 0.785 & 1.228 & 1.617 & 1.970 \\
Detergent & 0.957 & 1.007 & 1.020 & 1.019 & 1.012 \\
Disinfectant & 3.913 & 2.020 & 0.184 & -1.550 & -3.183 \\
Electric irons & 1.093 & 1.048 & 0.980 & 0.905 & 0.830 \\
Electric stoves & 0.140 & 0.874 & 1.303 & 1.607 & 1.843 \\
English apples & 0.916 & 0.995 & 1.032 & 1.053 & 1.066 \\
English potatoes & 1.046 & 1.029 & 0.992 & 0.949 & 0.905 \\
Evaporated milk & 0.952 & 0.999 & 1.019 & 1.029 & 1.034 \\
\hline & & & & & \\
\hline
\end{tabular}


Table 8. (Continued).

\begin{tabular}{|c|c|c|c|c|c|}
\hline Import category & $\begin{array}{c}\mathrm{S} 1 \\
(t=10 \%)\end{array}$ & $\begin{array}{c}\mathrm{S} 2 \\
(t=20 \%)\end{array}$ & $\begin{array}{c}\mathrm{S} 3 \\
(t=30 \%)\end{array}$ & $\begin{array}{c}\mathrm{S} 4 \\
(t=40 \%)\end{array}$ & $\begin{array}{c}\mathrm{S} 5 \\
(t=50 \%)\end{array}$ \\
\hline Flavoured preparations & 0.926 & 0.988 & 1.026 & 1.053 & 1.075 \\
\hline Flavoured syrups & 0.862 & 0.989 & 1.052 & 1.089 & 1.114 \\
\hline Flying fish & 0.858 & 0.971 & 1.047 & 1.108 & 1.159 \\
\hline Food preparations & 1.663 & 1.241 & 0.817 & 0.412 & 0.028 \\
\hline Gas stoves & 0.792 & 0.960 & 1.070 & 1.154 & 1.224 \\
\hline Grapefruits & 1.550 & 1.202 & 0.849 & 0.511 & 0.189 \\
\hline Grapes & 0.890 & 0.976 & 1.036 & 1.084 & 1.125 \\
\hline Green tea & -0.196 & 0.642 & 1.357 & 1.997 & 2.582 \\
\hline Herbicides & 0.952 & 1.000 & 1.019 & 1.028 & 1.032 \\
\hline Insecticides & 1.450 & 1.178 & 0.881 & 0.589 & 0.307 \\
\hline Jams & 1.075 & 1.044 & 0.985 & 0.919 & 0.851 \\
\hline Lettuce & 9.761 & 3.983 & -1.483 & -6.601 & -11.394 \\
\hline Liquid bleach & 1.047 & 1.032 & 0.992 & 0.946 & 0.898 \\
\hline Liquid detergent & 0.900 & 0.986 & 1.036 & 1.070 & 1.096 \\
\hline Luncheon meat & 0.984 & 1.009 & 1.010 & 1.002 & 0.990 \\
\hline Mackerels & 1.329 & 1.127 & 0.912 & 0.702 & 0.501 \\
\hline Malted beverages & -5.076 & -0.926 & 2.773 & 6.153 & 9.277 \\
\hline Margarine & 0.378 & 0.815 & 1.186 & 1.518 & 1.821 \\
\hline Mayonnaise & 0.997 & 1.014 & 1.006 & 0.990 & 0.971 \\
\hline Microwaves & 1.371 & 1.145 & 0.901 & 0.662 & 0.433 \\
\hline Mosquito coils & 0.831 & 0.960 & 1.054 & 1.133 & 1.201 \\
\hline Natural honey & 0.831 & 0.965 & 1.056 & 1.128 & 1.189 \\
\hline Oats & 1.153 & 1.074 & 0.965 & 0.849 & 0.734 \\
\hline Onions & 1.369 & 1.140 & 0.900 & 0.668 & 0.446 \\
\hline Orange juice & 1.426 & 1.162 & 0.885 & 0.616 & 0.360 \\
\hline Oranges & 0.909 & 0.988 & 1.033 & 1.063 & 1.085 \\
\hline Other biscuits & 0.864 & 0.973 & 1.045 & 1.101 & 1.148 \\
\hline Peanut butter & 0.534 & 0.860 & 1.139 & 1.389 & 1.618 \\
\hline Pepper sauce & 0.906 & 0.987 & 1.033 & 1.065 & 1.090 \\
\hline Pineapple juice & 1.952 & 1.339 & 0.735 & 0.162 & -0.380 \\
\hline Plantains & 0.763 & 0.941 & 1.075 & 1.187 & 1.286 \\
\hline Pork & 0.703 & 0.912 & 1.089 & 1.247 & 1.390 \\
\hline Portable radios & 1.012 & 1.019 & 1.002 & 0.977 & 0.950 \\
\hline Poultry feed & 1.072 & 1.036 & 0.983 & 0.928 & 0.873 \\
\hline Pumpkins & 1.600 & 1.225 & 0.837 & 0.462 & 0.104 \\
\hline Refrigerators & 1.040 & 1.042 & 0.999 & 0.941 & 0.879 \\
\hline Rice (bulk) & 1.177 & 1.079 & 0.956 & 0.831 & 0.708 \\
\hline Rice (pack) & 0.828 & 0.960 & 1.055 & 1.133 & 1.201 \\
\hline Room fans & 1.381 & 1.143 & 0.897 & 0.658 & 0.431 \\
\hline Salmon & 0.985 & 1.012 & 1.010 & 0.999 & 0.982 \\
\hline Salted pork & 1.066 & 1.041 & 0.988 & 0.927 & 0.864 \\
\hline Sardines & 0.971 & 1.006 & 1.014 & 1.013 & 1.007 \\
\hline Sauce (other) & 1.022 & 1.032 & 1.002 & 0.960 & 0.914 \\
\hline Sausages & 0.140 & 0.874 & 1.303 & 1.607 & 1.843 \\
\hline Scouring pads & 1.083 & 1.051 & 0.984 & 0.909 & 0.832 \\
\hline Scouring powder & 1.408 & 1.157 & 0.891 & 0.631 & 0.383 \\
\hline
\end{tabular}


Table 8. (Continued).

\begin{tabular}{lccccc}
\hline Import category & $\mathrm{S} 1$ & $\mathrm{~S} 2$ & $\mathrm{~S} 3$ & $\mathrm{~S} 4$ & $\mathrm{~S} 5$ \\
$(t=10 \%)$ & $(t=20 \%)$ & $(t=30 \%)$ & $(t=40 \%)$ \\
$(t=50 \%)$
\end{tabular}

used to predict product-level imports for 2007M1 to 2008M9. The calculated forecast evaluation statistics are provided in Table 6. All three forecast evaluation statistics suggest that the results of the disaggregated model were much closer to the actual values when compared to those from the pooled model. The Morgan-Granger-Newbold test (Granger and Newbold 1977), which evaluates the statistical significance of the two forecasts, suggests that this difference was also statistically significant at normal levels of testing.

Given that the model generates forecasts that are relatively close to actual values, the model is then employed to simulate the impact of tariff harmonisation on import demand as well as tariff revenues. Table 7 provides the deviation of the import values from the simulated baseline values. The baseline assumes that applied tariffs are set at the sample mean for tariffs over the period January 2000 to September 2008. Five tariff harmonisation scenarios are considered: (1) $10 \%$; (2) $20 \%$; (3) $30 \%$; (4) $40 \%$; and (5) $50 \%$. The results are provided in Table 7 . On average, the results suggest that as a result of harmonisation of applied tariffs to a level of $10 \%$, the demand for imports is likely to rise by about $10 \%$. In contrast, harmonisation to an applied tariff of $20 \%$ would lead to a mean increase in imports of $3 \%$. Tariff harmonisation at higher levels would lead to reductions in imported goods.

The impact of tariff harmonisation on tariff revenues is provided in Table 8. At the 10\% level, the rise in import demand more than offsets the reduction in tariff rates and therefore leads to a $1.2 \%$ increase in tariff 
duties. In contrast, tariff harmonisation at the $20 \%$ level resulted in a $2.3 \%$ expansion in tariff revenues relative to the baseline. Harmonisation of tariffs at the $30 \%$ level would essentially be revenue neutral, while rates any higher than this level would lead to a reduction in lower tax receipts.

\section{Conclusions}

Imports are very important to most industries in Caribbean economies. On average imports of goods and services represent about $64 \%$ of GDP for Caribbean countries, $37 \%$ percentage points above the average for the world. Understanding the factors that influence import demand in the Caribbean is crucial to the development of any foreign policy on the part in these countries (Lewis-Bynoe et al. 2002).

The present study uses disaggregated monthly observations on imports at the four-digit SITC level for 91 products between January 2000 and September 2008. The pooled import demand equation (which is similar to the traditional aggregate demand function that would normally be estimated elsewhere) had negative and statistically significant coefficients on the price and applied tariff variables. The null hypothesis that the income elasticity can be restricted to zero could not be rejected at normal levels of testing in this model. The coefficient on the relative price variable suggests that the demand for imports in Barbados is relatively price inelastic. Allowing the intercept to vary did not change the sign or significance of the elasticity of applied duties, albeit now less than unity. This suggests that tariff rate changes result in a less than proportionate response in the import volume demanded. Additionally, the income elasticity was now statistically different from zero, and had a coefficient greater than zero. This result implies that a $1 \%$ increase in income is likely to lead to a more than $1 \%$ rise in imports, and consequently the import to GDP ratio should climb over time.

The use of an F-test rejected the null hypothesis that the coefficients on relative prices, applied tariffs and income can be restricted across product groups. Thus, for policy simulations on possible tariff changes the disaggregated import demand equation was used. On average, the results suggested that as a result of harmonisation of applied tariffs to a level of $10 \%$, the demand for imports is likely to rise by about $10 \%$, while harmonisation to an applied tariff of $20 \%$ would lead to a mean increase in imports of $3 \%$. Tariff harmonisation at higher levels would lead to reductions in imported goods. Concomitantly, at the $10 \%$ level, the rise in import demand more than offsets the reduction in tariff rates and therefore leads to a $1.2 \%$ increase in tariff duties. Tariff harmonisation at the $20 \%$ resulted in a $2.3 \%$ expansion in tariff revenues relative to the baseline. Harmonisation of tariffs at the $30 \%$ would essentially be revenue neutral, while rates any higher than this level would lead to a reduction in lower tax receipts. 
In concluding, this study has demonstrated that there are definitely benefits to be accrued from the use of micro-econometric techniques to analyse issues surrounding import demand. However, we would argue that it is crucial that further research, expanding the basket of products be undertaken. Nonetheless, from this study it was identified that the best approach for Barbados, in light of talks on tariff harmonisation, would be an approach harmonising tariff to a level below or equal to the $30 \%$ level. Such attempts at harmonisation would enhance the process of administering duties, reduce administrative costs as well as enhance the ease of doing business. Administering duties is often somewhat contentious given product innovations and the invoicing practices of business. With a common tariff across all product groups, the ease of administering duties would be enhanced greatly. In a similar vein, given the simplicity of a single tariff system, it would be significantly easier to implement cost saving measures in regards to tax administration. Finally, having a single tariff would also reduce the uncertainty surrounding goods into the island and should therefore smooth the process of doing business.

\section{References}

Bahmani-Oskooee, Mohsen, and Hyun-Jae Rhee. 1997. Structural change in import demand behaviour, the Korean experience: A reexamination. Journal of Policy Modeling 19, no. 2: 187-93.

Baltagi, Badi H. 2005. Econometric analysis of panel data. West Sussex: John Wiley \& Sons Ltd. 55-6.

Birchwood, Anthony, and Julia Jhinkoo. 2007. The nexus between economic development and import demand in CARICOM economies. Paper presented at the 39th Annual Caribbean Centre for Monetary Studies Conference, Belize City, Belize.

Deyak, Timothy A., W. Charles Sawyer, and Richard L. Sprinkle. 1989. An empirical examination of the structural stability of disaggregated US import demand. Review of Economics and Statistics 71, no. 2: 337-41.

Gafar, John. 1995. Some estimates of the price and income elasticities of import demand for three Caribbean countries. Applied Economics 27, no. 11: 1045-8.

Granger, Clive W.J., and Paul Newbold. 1977. Forecasting economic series. New York: Academic Press.

Im, Kyung So, M. Hashem Pesaran, and Yongcheol Shin. 2003. Testing for units roots in heterogeneous panels. Journal of Econometrics 115, no. 1: 53-74.

James, Vanus. 2006. Import productivity growth, capital deepening and Caribbean development: Some theoretical insights. In Finance and real development in the Caribbean, ed. Anthony Birchwood, and Dave Seerattan, 223-36. Trinidad and Tobago: Caribbean Centre for Monetary Studies.

Levin, Andrew, Chien-Fu Lin, and Chia-Sang James Chu. 2002. Unit root test in panel data: Asymptotic and finite-sample properties. Journal of Econometrics 108, no. 1: 1-24.

Lewis-Bynoe, Denny, Jennifer Griffith, and Winston Moore. 2002. Trade liberalisation and the manufacturing sector: The case of a small developing country. Contemporary Economic Policy 20: 272-87. 
Mah, Jai Sheen. 1993. Structural change in import demand behaviour: The Korean experience. Journal of Policy Modeling 15, no. 2: 223-7.

Maxwell, Chanelle, and Winston Moore. 2004. External price competitiveness and trade in the Caribbean. Money Affairs 17, no. 2: 137-54.

Murray, Tracy, and Peter J. Ginman. 1976. An empirical examination of the traditional aggregate import demand model. Review of Economics and Statistics. 58, no. 1: 75-80.

Shiells, Clinton R. 1991. Errors in import-demand estimates based upon unit-value indexes. Review of Economics and Statistics 73, no. 2: 378-82.

Zietz, Joachim, and Donald K. Pemberton. 1993. Parameter instability in aggregate US import demand functions. Journal of International Money and Finance. 12, no. 6: 654-67. 